HUB-EP-97/40

SU-ITP-97-35

hep-th/9708065

\title{
Moving Moduli, Calabi-Yau Phase Transitions and Massless BPS Configurations in Type II Superstrings
}

\author{
Klaus Behrndt周 \\ Department of Physics, Stanford University \\ Stanford, CA 94305, USA

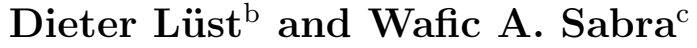 \\ Humboldt-Universität, Institut für Physik \\ Invalidenstraße 110, 10115 Berlin \\ Germany
}

\begin{abstract}
In this paper we discuss compactifications of type II superstrings where the moduli of the internal Calabi-Yau space vary over four-dimensional space time. The corresponding solutions of four-dimensional $N=2$ supergravity are given by charged, extremal BPS black hole configurations with non-constant scalar field values. In particular we investigate the behaviour of our solutions near those points in the Calabi-Yau moduli space where some internal cycles collapse and topology change (flop transitions, conifold transitions) can take place. The singular loci in the internal space are related to special points in the uncompactified space. The phase transition can happen either at spatial infinity (for positive charges) or on spheres (with at least one negative charge). The corresponding BPS configuration has zero ADM mass and can be regarded as a domain wall that separates topologically different vacua of the theory.
\end{abstract}

\footnotetext{
${ }^{\mathrm{a} e}$-mail: behrndt@qft2.physik.hu-berlin.de

be-mail: luest@qft1.physik.hu-berlin.de

${ }^{\mathrm{c}}$ e-mail: sabra@qft2.physik.hu-berlin.de
} 


\section{Introduction}

Recent developments showed that space-time geometry or space-time topology have no absolute meaning in string theory but are derived concepts which depend on how strings test their background spaces in various limits. For example, in the study of the moduli spaces of type II strings compactified on Calabi-Yau three-folds it became clear that the topologies of the Calabi-Yau spaces can be continuously deformed into each other; therefore many, or even possibly all, Calabi-Yau vacua are just branches of a larger universal moduli space. Calabi-Yau transitions, which were studied in the past, contain for example flop transitions where one moves through walls of the complexified Kähler moduli spaces (see for example [1], [2]) where the walls correspond to metrically degenerate Calabi-Yau spaces in which certain homologically nontrivial cycles have zero volume. Performing the flop transition the Calabi-Yau intersection numbers change, while the number of Kähler moduli is left unchanged. However the conformal field theory [3] stays perfectly well behaved when moving through a wall. Another transition is the conifold transition in the complex structure moduli space of type IIB superstrings on Calabi-Yau spaces. The conifold transitions [4] occur at those points in the moduli space where certain 3-cycles shrink to zero size and then are blown up as two cycles, changing in this way the Hodge numbers. However, unlike the previous case, the conifold transition cannot be described in the language of conformal field theory. The physical understanding of the conifold transition was provided by Strominger [5]; at the conifold point a BPS hypermultiplet black hole becomes massless, being responsible for the singularity in the moduli space metric of the $N=2$ vector multiplets at this point. The massless black can condense [6], i.e. acquire a non-vanishing vacuum expectation value (just in analogy to the monopole condensation in $N=2$ field theory [7]), and in this way one branches off into the moduli space of a Calabi-Yau space with different Hodge numbers $\left(h^{(1,1)}\right.$ gets larger, whereas $h^{(2,1)}$ gets reduced).

BPS saturated solutions of four-dimensional $N=2$ supergravity coupled to $N=2$ vector multiplets have been discussed in many recent papers [8, 9, 10, 11]. The simplest class of solutions is given by the double extreme $N=2$ black holes with non-vanishing electric and magnetic charges. For this type of solutions the values of the scalar moduli fields, which follow from a minimisation of the $N=2$ central charge, take constant values over the entire space-time. More general extremal supersymmetric black holes allow for nonconstant moduli fields, where the variation of the moduli fields over the four-dimensional space is provided by those harmonic functions which also determine the electric/magnetic field strengths of the charged black hole [10, 11]. So in this case of non-constant moduli, we have learned that the internal space does not decouple from the 4-dimensional space time. In particular the vector multiplet moduli, which determine the Käher moduli in type IIA Calabi-Yau compactifications or which, respectively, determine the sizes of the Calabi-Yau 3-cycles in the type IIB compactifications, vary over the uncompactified space in case of static extremal $N=2$ black hole solutions. Indeed one can argue that special or singular points in the internal space are related to special or singular points in space 
time (like horizons or curvature singularities). It is the aim of this paper to discuss this strong relationship.

After discussing the general $N=2$ supergravity solution, we will focus on the interplay between space time and internal space, which is assumed to be a Calabi-Yau-threefold of a type II compactification. First we will briefly study the variation of the type IIA Kähler moduli in the neighbourhood of the boundary of the complexified Kähler cone, i.e. for small value of a particular Kähler modulus. This discussion is relevant for the flop transition in four dimensions. Then we will more extensively discuss the type IIB conifold transition where one of the complex structure moduli fields is small. The following generic picture will emerge. If one moves through a non-singular space time one varies at the same time the radii of the cycles of the Calabi-Yau. At any point in space time the Calabi-Yau looks different. Any generic $N=2$ black hole has a non-singular horizon and this horizon in space time corresponds to an extremal radius of the Calabi-Yau. We will find that for vanishing 3-cycles in IIB compactifications, our solution contains massless BPS states.

\section{The self-dual 3-brane}

Before we come to the Calabi-Yau compactification, let us explain the picture for the torus compactification.

The self-dual 3-brane of type IIB string theory in 10 dimensions is given by

$$
\begin{gathered}
d s^{2}=\frac{1}{\sqrt{H}}\left(-d t^{2}+d z_{1}^{2}+d z_{2}^{2}+d z_{3}^{2}\right)+\sqrt{H}\left(d y_{1}^{2}+d y_{2}^{2}+d y_{3}^{2}+d x_{i}^{2}\right) \\
F=d \frac{1}{H} \wedge d t \wedge d z_{i}+{ }^{\star} d \frac{1}{H} \wedge d t \wedge d z_{i}
\end{gathered}
$$

where $i=1,2,3$ with the harmonic function $H$

$$
H=1+\frac{p}{r} \quad, \quad r^{2}=x_{i} x_{i}
$$

and $p$ is the charge. In general, the harmonic function can depend on all transversal coordinates $\left(y_{i}, x_{i}\right)$ yielding a non-singular space time. But since we are interested in a compactification to 4 dimensions, we assumed that $H$ is independent of all internal coordinates $\left(y_{i}, z_{i}\right)$, i.e. not only of world volume coordinates $\left(z_{i}\right)$ but also of the transversal coordinates $\left(y_{i}\right)$. This assumption makes already the 10-d solution singular. Let us look in more detail on this solution. Compactifying this solution means we wrap the 3-brane coordinates $\left(z_{i}\right)$ around one 3 -cycle and the remaining coordinates $\left(y_{i}\right)$ represent a second 3 -cycle. Both 3-cycles build the internal space, and their radii vary over space time. By approaching the singular point $r=0$ one 3-cycle diverges whereas the other vanishes.

The singularity in this case is a consequence of missing charges. In torus compactification we need at least 4 charges (or 4 branes) in order to obtain a non-singular black hole in 4 dimensions. The corresponding solution with four intersecting 3-branes is given by (we 
skip the gauge field part)

$$
\begin{aligned}
d s^{2}= & -\frac{1}{\sqrt{H_{1} H_{2} H_{3} H_{4}}} d t^{2}+\sqrt{H_{1} H_{2} H_{3} H_{4}} d x_{i} d x_{i}+ \\
& +\sqrt{\frac{H_{1} H_{2}}{H_{3} H_{4}}} d z_{1}^{2}+\sqrt{\frac{H_{3} H_{4}}{H_{1} H_{2}}} d y_{1}^{2}+\sqrt{\frac{H_{1} H_{4}}{H_{3} H_{2}}}\left(d z_{2}^{2}+d z_{3}^{2}\right)+\sqrt{\frac{H_{3} H_{2}}{H_{1} H_{4}}}\left(d y_{2}^{2}+d y_{3}^{2}\right) .
\end{aligned}
$$

$\left(H_{m}=1+p^{m} / r, m=1, \cdots, 4\right)$ and the branes are wrapped around the following internal coordinates

\begin{tabular}{lcccccc}
\hline & $z_{1}$ & $z_{2}$ & $z_{3}$ & $y_{1}$ & $y_{2}$ & $y_{3}$ \\
\hline$H_{1}-3-$ brane & & & & $\times$ & $\times$ & $\times$ \\
$H_{2}-3-$ brane & & $\times$ & $\times$ & $\times$ & & \\
$H_{3}-3-$ brane & $\times$ & $\times$ & $\times$ & & & \\
$H_{4}-3-$ brane & $\times$ & & & & $\times$ & $\times$ \\
\hline
\end{tabular}

where the world-volume coordinates are indicated by " $\times$ ". Now, all four 3-cycles stay finite when we approach the point $r=0$ and also the 4-d geometry is not singular at this point $\left(A d S_{2} \times S_{2}\right)$. But turning off one charge, e.g. $p^{1}$ (i.e. $\left.H_{1}=1\right)$, the configuration becomes singular, some 3 -cycles vanish whereas others diverge yielding a decompactification at $r=0$. This is typical for torus compactifications. It is not possible to shrink one cycle while keeping the others fixed.

The ADM mass for the solution (3) is

$$
M=\frac{1}{4}\left(p^{1}+p^{2}+p^{3}+p^{4}\right) .
$$

Hence, for a vanishing mass we need at least one negative charge. However, this yields a zero in the corresponding harmonic function $\left(H=1-\frac{|p|}{r}\right)$ at $r=|p|$. As a consequence one 3-cycle vanishes, but again the others diverge.

The situation changes completely when we compactify the 3-brane configuration on a Calabi-Yau threefold. Here, any generic solution has stabilized radii and a non-singular horizon. Even for vanishing 3-cycles, the other cycles remain non-singular (also the horizon), but the gauge couplings will diverge. It will be the subject of the next sections to address this question.

\section{Extremal black holes in $N=2$ supergravity}

We start with a short review of the general black hole solution of $N=2$ supergravity coupled to vector multiplets. Assuming that the scalar fields in the hyper multiplets are trivial, dhe supersymmetric solution for the black hole metric, the scalar field components $z^{A}$ and for the magnetic/electric fields strenths $G_{I m n}, F_{m n}^{I}$ is given by [10], [11]

$$
\begin{aligned}
& d s^{2}=-e^{2 U} d t+e^{-2 U} d x^{m} d x^{m} \quad, \quad z^{A}=\frac{X^{A}}{X^{0}} \\
& F_{m n}^{I}=\frac{1}{2} \epsilon_{m n p} \partial_{p} \tilde{H}^{I} \quad, \quad G_{I m n}=\frac{1}{2} \epsilon_{m n p} \partial_{p} H_{I},
\end{aligned}
$$

\footnotetext{
${ }^{\mathrm{d}}$ For solutions with non-trivial hyper multiplets see [12].
} 
with $m, n=1,2,3$ and

$$
\begin{aligned}
G_{I \mu \nu} & =\operatorname{Re} \mathcal{N}_{I J} F_{\mu \nu}^{J}-\operatorname{Im} \mathcal{N}_{I J^{\star}} F_{\mu \nu}^{J}, \\
e^{-2 U}=e^{-K} & \equiv i\left(\bar{X}^{I} F_{I}-X^{I} \bar{F}_{I}\right),
\end{aligned}
$$

where $K$ is the Kähler potential of the moduli space metric, and the holomorphic section $\left(X^{I}, F_{I}\right)$ are constrained by

$$
i\left(X^{I}-\bar{X}^{I}\right)=\tilde{H}^{I}\left(x^{\mu}\right) \quad, \quad i\left(F_{I}-\bar{F}_{I}\right)=H_{I}\left(x^{\mu}\right) .
$$

Since the symplectic vector $\left(\tilde{H}^{I}, H_{I}\right)$ is introduced via the gauge fields, this condition can be seen as a relation between the holomorphic section $\left(X^{I}, F_{I}\right)$ and the gauge field section $\left(F_{\mu \nu}^{I}, G_{\mu \nu I}\right)$. As a consequence of the Bianchi identities, the $H^{\prime} s$ have to be harmonic and for the single-center case we take

$$
\tilde{H}^{I}=\tilde{h}^{I}+\frac{p^{I}}{r} \quad, \quad H_{I}=h_{I}+\frac{q_{I}}{r}
$$

where $q_{I}$ and $p^{I}$ are the electric and magnetic charges carried by the black hole. The constant parts determine the scalar fields at infinity and parameterize the moduli space.

In addition, we have the two constraints

$$
\left(H_{I} \partial_{m} \tilde{H}^{I}-\tilde{H}^{I} \partial_{m} H_{I}\right)=0 \quad, \quad e^{-2 U_{\infty}}=1 .
$$

The first condition ensures that the Kähler connection vanishes and is necessary for any static solution (see [10], 11]) and the second one fixes our coordinate system. These two conditions fix two of the $h^{\prime} s$ and we have the total number of $2 n$ continuous variable parameterizing the $2 n$ dimensional moduli space.

In order to discuss the singularities of the black hole metric (6) we compute the square of the space-time curvature tensor

$$
\begin{gathered}
R_{\mu \nu \rho \sigma} R^{\mu \nu \rho \sigma}=\frac{1}{V^{8}}\left(32\left(\partial_{m} V \partial_{m} V\right)^{2}-48 V \partial_{n} \partial_{m} V \partial_{m} V \partial_{n} V-2 V \partial_{m} V \partial_{m} V \partial_{n} \partial_{n} V+\right. \\
\left.+8 V^{2} \partial_{n} \partial_{m} V \partial_{n} \partial_{m} V+V^{2}\left(\partial_{m} \partial_{m} V\right)^{2}\right),
\end{gathered}
$$

where $V=e^{-U}=e^{-K / 2}$.

\section{The solution near a wall in the Calabi-Yau Kähler moduli space}

In this section we want to construct the black hole solutions in the limit where one of the $h^{(1,1)}$ Kähler moduli $z^{A}\left(A=1, \ldots, h^{(1,1)}\right)$ in type IIA compactifications on a Calabi-Yau space becomes small. These moduli correspond to sizes of 2-cycles, respectively of the 
Hodge dual 4-cycles in the Calabi-Yau threefold; hence they must be positive defining the Kähler cone. If one of the Kähler moduli becomes small the Calabi-Yau space degenerates in one of the following three ways [2]

(i) A two cycle collapses to a point.

(ii) A complex divisor collapses either to a curve or to a point.

Case (i) corresponds to a topology change via a flop transition between two different but birationally equivalent Calabi-Yau spaces, where by moving through the wall a new geometrical Kähler cone is reached; the union of all geometrical Kähler cones is called the extended Kähler cone. During the flop transition one Kähler modulus, say $z^{2}$ changes its sign and the size of the new 2-cycle is given by $-z^{2}$. This has the effect that the intersection numbers change by the new term $C_{222}=-\frac{1}{6}$ [13]. (Several concrete examples of flop transitions in particular Calabi-Yau spaces where investigated in [14].) However in four-dimensional type IIA compactifications on a Calabi-Yau space, the flop transition is in fact not a sharp transition since one can turn on the axionic components of the complex moduli fields. We will discuss in the following the case of vanishing axions. Moreover in four-dimensions there are in general non-geometrical phases outside the extended Kähler cone due to the effect of world sheet instantons. In the following we will neglect all effects coming from world sheet instantons. This resembles in a way the decompactification to five dimensions or $M$-theory on a Calabi-Yau manifold [15], since in five dimensions the axions fields are frozen and no non-geometrical phases exist [2] due to the absence of world sheet instantons. Phase transitions via flops in 5-dimensional compactifications with constant moduli fields were recently discussed in [16].

Now let us discuss the extremal $N=2$ black holes for small values of the Kähler moduli. At the vicinity of the Kähler cone wall, i.e. for small Kähler moduli, we expand the $N=2$ prepotential as

$$
F\left(X^{I}\right)=\left(X^{0}\right)^{2}\left[-\frac{1}{6} C_{A B C} z^{A} z^{B} z^{C}-\frac{i \chi \zeta(3)}{2(2 \pi)^{3}}\right],
$$

where the $z^{A}=X^{A} / X^{0}$ are the Kähler moduli and the $C_{A B C}$ are the classical intersection numbers. Assuming that $z^{2}$ corresponds to the vanishing cycle, the intersection numbers change via the flop transition

$$
-\frac{1}{6} C_{A B C} z^{A} z^{B} z^{C} \rightarrow-\frac{1}{6} C_{A B C} z^{A} z^{B} z^{C}+\frac{1}{6}\left(z^{2}\right)^{3}
$$

Next, we have to solve the constraints (8). An easy way to find solutions is to restrict ourselves to the axion-free case, i.e.

$$
\operatorname{Re} z^{A}=0
$$

and find as a solution

$$
X^{0}=\frac{\lambda}{2} \quad, \quad X^{A}=-i \frac{\tilde{H}^{A}}{2} \quad \text { and } \quad z^{A}=-i \frac{\tilde{H}^{A}}{\lambda} .
$$


So we are dealing with a black hole with electric charge $q_{0}$ and non-vanishing magnetic charges $p^{A}$. The function $\lambda$ is fixed by the equation $F_{0}-\bar{F}_{0}=-i H_{0}$. Expanding the solution of this cubic equation in powers of the intersection form we find

$$
\lambda=\frac{H_{0}}{2 c}+\frac{2 c}{\left(H_{0}\right)^{2}}\left(\frac{1}{6} C_{A B C} \tilde{H}^{A} \tilde{H}^{B} \tilde{H}^{C}\right) \pm . .
$$

with $c=\chi \zeta(3) / 2(2 \pi)^{3}$. Inserting this into $e^{-2 U}$ yields

$$
e^{-2 U}=\frac{\left(H_{0}\right)^{2}}{4 c}-\frac{3 c}{H_{0}}\left(\frac{1}{6} C_{A B C} \tilde{H}^{A} \tilde{H}^{B} \tilde{H}^{C}\right) \pm . .
$$

Following the procedure, e.g., described in the last reference of [9], one could easily calculate further corrections to the prepotential (12). For a microscopic discussion see also the first ref. of [9].

For this axion-free solution, a vanishing cycle $\left(z^{2}=0\right)$ corresponds to a vanishing harmonic function $\left(\tilde{H}^{2}=0\right)$, see (15). With $\tilde{H}^{2}=\tilde{h}^{2}+\frac{p^{2}}{r}$, it follows that the relevant cycle is vanishing at the special radius $r=-\frac{p^{2}}{\tilde{h}^{2}}$. So the charge $p^{2}$ must be negative $\left(\tilde{h}^{2}>0\right)$. Crossing this critical radius the flop transition takes place and $z^{2}$ becomes negative. We see that, even if the intersection form vanishes on the Kähler wall $z^{2}=0$ the $H_{0}$ part regularizes the solution, i.e. the space-time black hole metric is regular on the wall of the Kähler cone. When going through the wall, we have to take into account the change in the intersection numbers as given in (13) in order to get the solution on the other side. In contrast to the conifold transition discussed below, the Hodge numbers do not change in this transition. Thus, the number of vector multiplets is the same. In the next section we will also discuss the possibility where the phase transition takes place at spatial infinity.

Our solution (15) with magnetic charges $p^{A}$ has a nice brane interpretation in $M$-theory. It consists of an intersection of three $M 5$-brane, which have a common string. The remaining 4 directions are wrapped around 4-cycles of the Calabi Yau space and the charges indicate how many times the 5-branes are wrapped. Every of these 4-cycles is parameterized by one harmonic function. In 5 dimensions we obtain a magnetic string and $H_{0}$ parameterizes the momentum modes traveling along this string. In 11 dimensions these momentum modes correspond to gravitational waves, i.e. pure gravity solution. It is interesting, that this gravity part regularizes our solution, even for vanishing intersection part. Since the constant $c$ is essentially the Euler number, this contribution is related to $R^{4}$ terms of the $M$-theory, for a recent discussion see [17]. Hence, the regularization of the 4 -d black hole is caused by higher curvature terms in 11 dimensions.

\section{The solution near a Calabi-Yau conifold point}

A Calabi-Yau space has $b_{3}=2 h^{(2,1)}+2$ topologically non-trivial 3-cycles. One introduces a basis of 3 -cycles $\left\{\gamma_{I}, \delta^{J}\right\}\left(I, J=0 \cdots h^{2,1}\right)$ such that

$$
\gamma \cap \delta=-\delta \cap \gamma=\mathbf{1}, \gamma \cap \gamma=\delta \cap \delta=0
$$


In terms of the holomorphic 3 -form $\Omega$, the corresponding periods are

$$
F_{I}=\int_{\gamma_{A}} \Omega \quad, \quad X^{I}=\int_{\delta^{I}} \Omega
$$

where $X^{I}$ can be used as projective coordinates on the moduli space $\mathcal{M}$ of complex structures. So the parameters for the complex structure moduli can be defined as $z^{A}=$ $X^{A} / X^{0}\left(A=1 \cdots, h^{(2,1)}\right)$.

The conifold point is in general described by a locus of co-dimension $k$ in $\mathcal{M}$, where $k$ cycles $X^{L}(L=1 . . k)$ vanish, while the remaining cycles stay finite. In the following we will discuss the most simple situation with periods $X^{0}$ and $X^{1}$ (together with $F_{0}$ and $F_{1}$ ), where $X^{1}$ vanishes at the conifold point and $X^{0}$ remains finite (there might be other periods, but they do not influence the results). This captures all the interesting physics and can be easily generalised to the higher dimensional case. So at the conifold point we have

$$
z^{1}=0 \quad \text { or }: \quad \operatorname{Im} z^{1}=\operatorname{Re} z^{1}=0 .
$$

In addition, if the 3 -fold is transported about a closed loop around the singular surface the period $F_{1}$ undergoes a monodromy transformation [6]

$$
X^{1} \rightarrow X^{1} \quad, \quad F_{1} \rightarrow F_{1}+X^{1} .
$$

Therefore, near this point the prepotential can be expanded as

$$
F=\left(X^{0}\right)^{2} \mathcal{F}=-i\left(X^{0}\right)^{2}\left(c+\frac{1}{4 \pi}\left(z^{1}\right)^{2} \log i z^{1}+(\text { analytic terms })\right) .
$$

The constant $c$ contains, e.g., the Euler number $\chi$ of the Calabi-Yau $\left(c=\chi \zeta(3) / 2(2 \pi)^{3}\right)$. With eq.(17) it is easy to see that the Kähler potential is finite at the conifold point:

$$
e^{-K}=4\left|X^{0}\right|^{2} \frac{\chi \zeta(3)}{2(2 \pi)^{3}}
$$

This will mean that the space-time curvature square eq.(11) at the conifold point will also be finite. In contrast, the internal moduli space metric diverges at the conifold point.

Next, let us look at the structure of the space-time solution near this point. Assuming that $X^{0}$ is not zero or divergent (to keep all other cycles finite), it follows that at the conifold point

$$
\operatorname{Re} X^{1}=0 \quad, \quad 2 \operatorname{Im} X^{1}=\tilde{H}^{1}=0 .
$$

For simplification, we will consider axion-free black holes. Thus, we assume

$$
\operatorname{Re} z^{1} \equiv 0
$$

and take as solution of (8)

$$
X^{0}=\frac{\lambda}{2} \quad, \quad X^{1}=-i \frac{\tilde{H}^{1}}{2}
$$


where the function $\lambda$ is fixed by

$$
i\left(F_{0}-\bar{F}_{0}\right)=H_{0}=2 c \lambda+\frac{\left(\tilde{H}^{1}\right)^{2}}{4 \pi \lambda} .
$$

Since $\lambda$ should stay finite, otherwise the non-vanishing cycles would decompactify, we take for $\lambda$ the solution

$$
\lambda=\frac{H_{0}}{2 c}-\frac{\left(\tilde{H}^{1}\right)^{2}}{4 \pi H_{0}}+\mathcal{O}\left(\left(\tilde{H}^{1}\right)^{4}\right) .
$$

Keeping only the first correction, we obtain for the function $e^{-2 U}$ in the metric (6)

$$
\begin{aligned}
e^{-2 U} & =i\left(\bar{X}^{I} F_{I}-X^{I} \bar{F}_{I}\right)=\frac{\lambda}{2} H_{0}+\frac{\left(\tilde{H}^{1}\right)^{2}}{4 \pi}\left(\log \frac{\tilde{H}^{1}}{\lambda}+\frac{1}{2}\right) \\
& =\frac{H_{0}^{2}}{4 c}+\frac{\left(\tilde{H}^{1}\right)^{2}}{4 \pi} \log \frac{2 c \tilde{H}^{1}}{H_{0}} \pm . .
\end{aligned}
$$

which is non-singular/non-vanishing even at points where the 3 -cycle vanish $\left(\tilde{H}^{1}=0\right)$. Already the $X^{0}$ part in the prepotential regularises the metric. Taking into account further analytic terms from the expansion (22) will not alter this qualitative picture. But note, because of the logarithm we cannot extend the solution beyond this point, i.e. formally to negative $\tilde{H}^{1}$. Instead, $\tilde{H}^{1}$ remain zero and new functions will appear that parameterise the new emerging cycles.

Again keeping only the first correction, our periods at infinity are given by

$$
\begin{aligned}
& X_{\infty}^{0}=\frac{h_{0}}{4 c}-\frac{\left(\tilde{h}^{1}\right)^{2}}{8 \pi h_{0}} \quad, \quad X_{\infty}^{1}=-i \frac{\tilde{h}^{1}}{2} \\
& F_{0}^{\infty}=-i\left(\frac{h_{0}}{2}+\frac{c\left(\tilde{h}^{1}\right)^{2}}{8 \pi h_{0}}\right) \quad, \quad F_{1}^{\infty}=-\frac{\tilde{h}^{1}}{4 \pi}\left(\frac{1}{2}+\log \frac{2 c \tilde{h}^{1}}{h_{0}}\right) .
\end{aligned}
$$

The black hole carries electric $\left(q_{0}\right)$ and magnetic $\left(p^{1}\right)$ charges, see (8) and (9), and its mass can be read off from the metric eq.(29) and can be written in the usual way

$$
M=\left|q_{0} X_{\infty}^{0}-p^{1} F_{1}^{\infty}\right|
$$

Taking into account more analytic terms in the prepotential (22) would correspond to additional magnetic charges. Note, $h_{0}$ does not define a modulus, it is fixed by the second constraints in (10); the first one is identically fulfilled for axion-free black holes. On the other hand, $\tilde{h}^{1}$ is a modulus. In order to justify the expansion of the prepotential eq.(22) around the conifold point, $\tilde{h}^{1}$ should be small.

In order to make the picture complete we have to discuss the options for a vanishing harmonic function, i.e. to fulfill equation (24). We discussed already one possibility for the toroidal case, namely to take negative charges. This means

$$
\tilde{H}^{1}=\tilde{h}^{1}-\frac{\left|p^{1}\right|}{r} \text {. }
$$


Therefore, for the special radius $r=\frac{\left|p^{1}\right|}{\tilde{h}^{1}}=-i \frac{\left|p^{1}\right|}{2 X_{\infty}^{1}}$ (i.e. on an $S_{2}$ sphere) the 3-cycles collapses and at special points in moduli space, where

$$
q_{0} X_{\infty}^{0}=-F_{1}^{\infty}\left|p^{1}\right|
$$

the BPS state (black hole) becomes massless (note $F_{1}^{\infty}$ is negative). But if this relation is not fulfilled the black hole is still massive. This seems to be in contradiction to Strominger's statement [5] that a vanishing cycle is always related to a massless black hole. The reason is that we still kept $q_{0}$ as independent charge, i.e. we still have a bound state of 2 objects. Setting this charge to zero, the mass vanish iff $F_{1}^{\infty} \sim \tilde{h}^{1}=0$ for arbitrary magnetic charge. Doing this $\left(q_{0}=\tilde{h}^{1}=0\right)$ in $(\sqrt[29]{)}$, we obtain as massless black hole solution

$$
e^{-2 U}=1+\frac{\left(p^{1}\right)^{2}}{8 \pi r^{2}} \log \frac{c\left(p^{1}\right)^{2}}{r^{2}} \pm . . \quad, \quad z^{1}=-i \frac{\sqrt{c} p^{1}}{r(1 \mp . .)} \quad, \quad F_{m n}=\epsilon_{m n p} \partial_{p} \frac{p^{1}}{r} .
$$

where we used $h_{0}=2 \sqrt{c}$, in order to have an asymptotic Minkowski space and $\pm \cdots$ indicate higher powers in $1 / r$. The exact solution is given by (29), if one inserts the exact solution for $\lambda$ from eq. (27). This is dual to the electric configuration discussed by Strominger. In order to keep the sign of $z^{1}$, the charge $p^{1}$ has to be positive.

To our knowledge, this solution has not been discussed before. Therefore, let us add some further comments. The main property of this massless solution is that it carries only one charge and has a shrinking internal 3 -cycle at spatial infinity $\left(z^{1} \rightarrow 0\right.$ for $\left.r \rightarrow \infty\right)$. We have expanded the solution around this vanishing cycle, but what about the black hole singularity/horizon? To discuss this question we have to approach the point $r=0$. Since $X^{1}$ blows up in this limit, our expansion breaks down and we have to take a different expansion in order to find the new parameter $\lambda$. The main contribution in this region will come from the cubic intersection part and as solution near $r=0$ one obtains $\lambda \sim \sqrt{\left(\tilde{H}^{1}\right)^{3} / H_{0}}$. Keeping in mind that $H_{0}=2 \sqrt{c}$ for this solution, we find $\exp \{-2 U\} \sim\left(\tilde{H}^{1}\right)^{3 / 2}$, i.e. this solution has a singular horizon (vanishing area). This however, is a typical example of a compactification singularity. In the mirror-mapped IIA solution, this black hole appears as compactification of the 5-d magnetic string, which is a wrapped M5-brane. Turning of the electric charges (see before eq. (34)), means that there are no momentum modes traveling along this string and after compactification it becomes a singular black hole (see also the next footnote).

\section{Summary and discussion}

The aim of this paper was to discuss the strong relationship between the 4-dimensional space time and the internal space. The general picture is shown in figure 1: for general extremal $N=2$ black hole solutions the moduli, i.e. the cycles of the internal CalabiYau space vary over space-time. We found two solutions, describing Calabi-Yau phase transitions. In the first solution, at special radii in (uncompactified) space the CalabiYau degenerates and topology change can occur. These special radii can be seen as the 


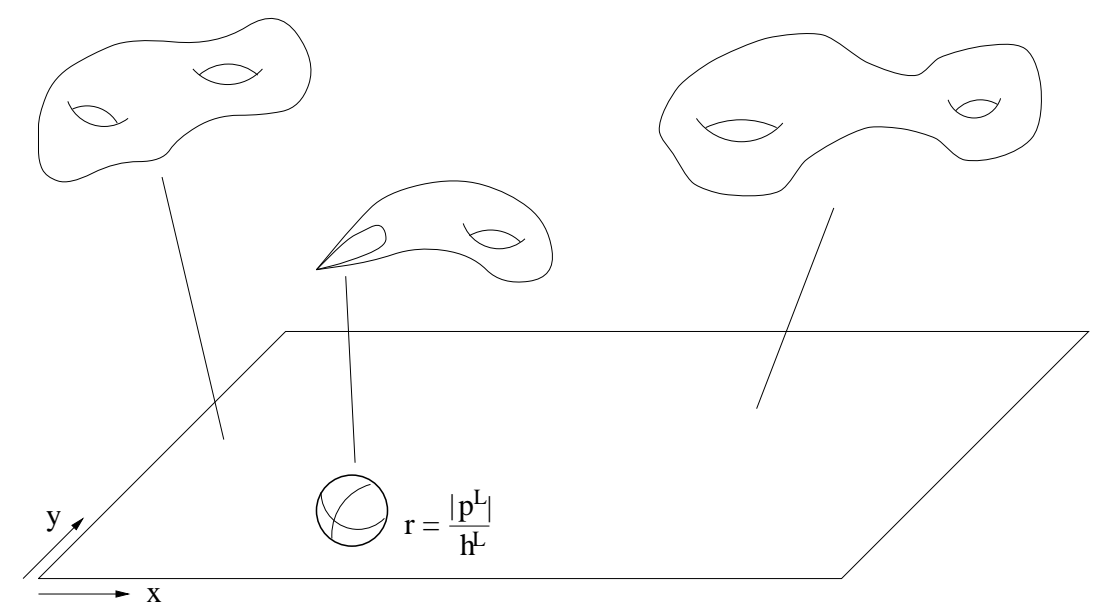

Figure 1: This figure shows the Calabi-Yau space varying over the three-dimensional space. In type IIB strings, at the conifold point some 3-cycles of the Calabi-Yau space vanish and are replaced by topologically different cycles. We argue, that this happens on $S_{2}$ spheres in space time where harmonic functions vanish. These spheres can be seen as domain walls that separate topological different vacua.

positions of the massless extremal black holes. This solution carries two charges, where one of them has to be negative. The second solution is described by only one positive charge and the phase transition point is at spatial infinity. This solution represents a (so far unknown) massless black hole.

What can we say about the black hole singularity/horizon? For torus compactification the space-time solution becomes singular at the internal degeneration loci (see eq. (3)). In this case, any vanishing cycle is accompanied by diverging cycles (decompactification). However, for Calabi-Yau compactification, the black hole metric stays finite at the loci of collapsing cycles. One reason why the black holes are non-singular in Calabi-Yau compactifications is that in contrast to the torus compactification, the branes are wrapped in a topological non-trivial manifold, which especially means that a brane can intersect with itself. For the 4-d black hole these self-intersections have the same consequence as intersection of different branes - the black hole becomes less singular. This stabilisation due to brane-intersection especially applies for the flop transition in type IIA compactifications. As long as one keeps $H_{0}$ non-trivial, , any 10-d configuration yields a non-singular black hole upon Calabi-Yau compactification. Near the horizon we always get the non-singular Reissner-Nordstrøm solution with stabilised scalars. This is true as long as we restrict ourselves on the static case. For rotating black holes one has a ring singularity and these arguments do not hold. This coincides with the solutions discussed in this paper. Only the single charged solution, describing the phase transition at infinity, is singular at $r=0$,

${ }^{\text {e}}$ The special role of $H_{0}$ can be understood by looking on the IIA side. It parameterises KK modes related to the $S_{1}$ in $M$ theory compactification. This circle cannot be stabilised by effects coming from the Calabi-Yau and turning off these KK-modes, the 4-d black hole becomes singular, e.g. the black hole (34). 
since $H_{0}$ is trivial in this case. The other solutions remain finite, even at points where the internal cycle collapses.

Another interesting question is what happens if we go beyond the special radius in space where the Calabi-Yau degenerates. For the type IIA flop transition it seems that there is no problem for the modulus, i.e the harmonic function to become negative beyond the wall; namely one is just entering a new Kähler cone. However, for the conifold transition, it is not so clear how to continue our solutions beyond the special radius, since at the conifold point the Hodge numbers change, and new harmonic functions emerge that correspond to the new cycles.

It is even not quite clear whether one should call our solutions black holes. Instead, it seems attractive to see these objects (spheres) as domain walls that separate two (topologically) different vacua of the theory. Since they are massless, they do not contribute to the total ADM mass. For the torus compactification these spheres are singular $\left(R_{\text {curv }}^{2} \sim(r-|p|)^{-8}\right.$ for two negative charges), however Calabi-Yau corrections smooth out the singularity (see (29)). So, if one would know the solution from "the inside" it should

be possible to connect the two regions. In this sense the massless states are a "door" to another world.

\section{Acknowledgments}

We thank G. Curio and T. Mohaupt for useful conversations. K.B. thanks John Schwarz and the group at Caltech for useful discussions. In addition, he would like to thank the Stanford University for its hospitality. The work is supported by the Deutsche Forschungsgemeinschaft (DFG) and by the European Commision TMR programme ERBFMRXCT96-0045. W. A. S is partially supported by DESY-Zeuthen.

\section{References}

[1] P. Aspinwall, B. Greene and D. Morrison, Nucl. Phys. B416 (1994) 414; hep-th/9309097.

[2] E. Witten, Nucl. Phys. B471 (1996) 195, Nucl. Phys. B471 (1996) 195; hep-th/9603150.

[3] E. Witten, Nucl. Phys. B403 (1993) 159, hep-th/9301042.

[4] C.H. Clemens, Adv. Math. 47 (1983) 107; R. Friedman, Math. Ann. 274 (1986) 671; F. Hirzebruch, "Some Examples of Threefolds with Trivial Canonical Bundle", in Gesammelte Abhandlungen, Band II, Springer-Verlag (1987) pp 757;

P. Candelas and X. de la Ossa, Nucl. Phys. B342 (1990) 246;

P. Candelas, P. Green and T. Hübsch, Nucl. Phys. B330 (1990) 49;

P. Candelas, X. de la Ossa, P. Green and L. Parkes, Nucl. Phys. B359 (1991) 21. 
[5] A. Strominger, Nucl. Phys. B451 (1995) 96, hep-th/9504090.

[6] B. Greene, D. Morrison and A. Strominger, Nucl. Phys. B451 (1995) 109, hep-th/9504145.

[7] N. Seiberg and E. Witten, Nucl. Phys. B426 (1994) 19, hep-th/9407087.

[8] S. Ferrara, R. Kallosh and A. Strominger, Phys. Rev. D52 (1995) 5412, hepth/9508072;

S. Ferrara and R. Kallosh, Phys. Rev. D54 (1996) 1514, hep-th/9602136;

S. Ferrara, R. Kallosh Phys.Rev. D54 (1996) 1525, hep-th/9603090;

R. Kallosh, M. Shmakova and W.K. Wong, Phys. Rev. D54(1996) 6284, hepth/9607077;

S. Ferrara, G.W. Gibbons, R. Kallosh, Black holes and critical points in moduli space, hep-th/9702103;

K. Behrndt, R. Kallosh, J. Rahmfeld, M. Shmakova and W.K. Wong, Phys. Rev. D54 (1996) 6293, hep-th/9608059;

G. Lopes Cardoso, D. Lüst and T. Mohaupt, Phys. Lett. B388 (1996) 266, hep-th/9608099;

K. Behrndt, G. Lopes Cardoso, B. de Wit, R. Kallosh, D. Lüst and T. Mohaupt, Nucl. Phys. B488 (1997) 236, hep-th/9610105.

[9] K. Behrndt, T. Mohaupt, Entropy of N=2 black holes and their M-brane description, hep-th/9611140;

K. Behrndt, G. Lopes Cardoso, I. Gaida, Quantum N=2 supersymmetric black holes in the $S$-T model, ep-th/9704095.

[10] W. A. Sabra, General static $N=2$ black holes, hep-th/9703101;

W. A. Sabra, Black holes in $N=2$ supergravity and harmonic functions, hep-th/9704147.

[11] K. Behrndt, D. Lüst and W.A. Sabra, Stationary solutions of N=2 supergravity, hep-th/9705169.

[12] K. Behrndt, I. Gaida, D. Lüst, S. Mahapatra and T. Mohaupt, From Type IIA Black Holes to T-dual Type IIB D-Instantons in $N=2, D=4$ Supergravity, hep-th/9706096.

[13] I. Antoniadis, S. Ferrara and T.R. Taylor, Nucl. Phys. B460 (1996) 489, hep-th/9511108.

[14] J. Louis, K. Sonnenschein, S. Theisen and S. Yankielowicz, Nucl. Phys. B480 (1996) 185; hep-th/9606049.

[15] S. Ferrara, R.R. Khuri and R. Minasian, Phys. Lett. B375 (1996) 81; hep-th/9602102. 
[16] A. Chou, R. Kallosh, J. Rahmfeld, S.-J.Rey, M. Shmakova and W. Kai Wong, Critical points and phase transitions in 5-D compactifications of $M$ theory, hep-th/9704142

[17] I. Antoniadis, S. Ferrara, R. Minasian and K.S. Narain, $R^{4}$ Couplings in $M$ and type II theories on Calabi Yau spaces, hep-th/9707013. 